\title{
Using Mobile Augmented Reality to Improve Attention in Adults with Autism Spectrum Disorder
}

\author{
Katherine Wang \\ University College London \\ London WC1E 6BT, UK \\ katherine.wang.19@ucl.ac.uk \\ Bingqing Zhang \\ University College London \\ London WC1E 6BT, UK \\ bingqing.zhang.18@ucl.ac.uk

\section{Youngjun Cho} \\ University College London \\ London WC1E 6BT, UK \\ youngjun.cho@ucl.ac.uk
}

Permission to make digital or hard copies of part or all of this work for personal or classroom use is granted without fee provided that copies are not made or distributed for profit or commercial advantage and that

copies bear this notice and the full citation on the first page. Copyrights for third-party components of this work must be honored. For all other uses, contact the owner/author(s)

Uses, contact the owner/author(s).

(c) 2020 Copyright is held by the owner/author(s).

(C) 2020 Copyright is held by the owner/

ACM ISBN 978-1-4503-6819-3/20/04.

\begin{abstract}
Adults on the autism spectrum commonly experience impairments in attention management that hinder

many other cognitive functions necessary to appreciate relationships between sensory stimuli. As autistic

individuals generally identify as visual learners, the effective use of visual aids can be critical in developing

life skills. In this brief paper, we propose a Mobile Augmented Reality for Attention (MARA) application which addresses a lack of supportive and simple costeffective solutions for autistic adults to train attention management skills. We present the proposed design, configuration and implementation. Lastly, we discuss future directions for research.
\end{abstract}

\section{Author Keywords}

Assistive technology; autism; attention; augmented reality; mobile applications.

\section{CSS Concepts}

- Human-centered computing Human computer interaction (HCI); Interaction paradigms Mixed /

augmented reality 


\section{Introduction}

Autism Spectrum Disorder (ASD) is a pervasive neurodevelopmental disorder characterized by earlyonset impairments in social communication, restricted or repetitive behavior [2] and attention management [32]. This has a long-term effect in adulthood in a large number of population $[7,11]$ (e.g. $1 \%$ of the total population in the UK in 2010 [8]). Studies have shown that attention management is critical to the performance of other cognitive functions (e.g. decision-making, memory, problem solving [16]). Relationships between different types of attention (see Table 1) and sensory-processing abilities $[11,25]$ have also been demonstrated. For instance, over-arousal to sensory information often occurs with over-selective attention, and is commonly exhibited in ASD [13]. This, in turn, is closely related to subsequent displacement behaviors in response to moderate arousal levels (e.g. hand-flapping, hyper-focused concentration on a single detail) [25].

Attention function impairments imply individuals with ASD may not perceive and integrate complex stimuli [14]; therefore, individuals may not be able to appreciate relationships between them. This disability has been shown to hinder many aspects of life for adults impacted by it (e.g., social communication, learning). For instance, few autistic adults develop and maintain the necessary skills to reach successful independent living [39].

Autistic individuals excel at visual search compared to neurotypical individuals and are most effective at processing visual stimuli compared to other sensory stimuli $[20,28]$. Given this, interventions have been developed on this basis, using either real objects, photographs, words, line drawings, or dynamic stimuli to help ameliorate social and behavioral impairments $[28,36]$. Technology, such as hand-held devices, has often been exploited, playing a key role in addressing ASD-related challenges [3]. Amongst other

technological intervention strategies, augmented reality (AR) has been shown to be effective and more engaging given the unique way in which combinations of different types of visual representations are assimilated and understood more easily than non-visual cues $[3,6,23]$. This can also enhance real-world interactions by facilitating exposure to complex and dynamic stimuli through controlled virtual environments. AR on mobile devices can allow for costeffective evaluation of symptomatic behaviors $[16,33]$. This explains its growing popularity for therapeutic and supportive uses.

Increasing efforts are being devoted to non-institutional rehabilitation support for autistic adults to develop adaptive skills to facilitate a sense of independence $[17,23]$. However, the presence of external barriers (e.g., inadequate access to technology, clinical support, and finances) and internal limitations, such as distraction and frustration autistic adults are susceptible to facing [21], restrict the use of assistive technology as an effective rehabilitative tool. The aim of this paper is to propose a system that is easy to use and inexpensive for autistic adults to improve attention management skills by interacting with real-world objects. The evolving capabilities of AR to merge reality with virtual elements in meaningful ways motivates us to explore the potential for its usage in developing novel interventions. 
The key contribution of this work is a novel and simple design of a mobile AR attention training system as a cost-effective self-help intervention to support adults on the autism spectrum to train attention management. This builds on our literature review on existing studies on the feasibility of AR as a support tool for ASD. Next, we describe the design strategy and implementation of our proposed AR application. Finally, we discuss directions for future work.

\section{Related Work}

Different AR functions have been used to enhance interventions for people with ASD in a variety of ways. For instance, visual cues are used to teach social skills $[24,26,30]$. Gamification is used to elicit pretend play $[4,37]$, mapping technology is used to train facial recognition $[29,38]$, and trigger-based modeling is used for education purposes to teach vocabulary [27] and reading [18]. AR-based intervention differentiates itself from traditional interventions in its ability to bridge the gap between physical and virtual worlds[26]. Also, Bai et al. [4] highlighted higher engagement in AR intervention in comparison with non-AR.

Most research has focused on childhood interventions in improving ASD-inflicted attention management. For instance, smart-glasses were used to provide visual cues in the form of arrows to guide a child's gaze to a facilitator's face, which was overlaid with a virtual mask to further attract attention. This resulted in decreases in measures of inattention, as well as hyperactivity and impulsivity, 48 hours after the intervention [40]. Also, mobile AR has been used to increase the effectiveness of such interventions given its engaging and costeffective way where focus can be controlled $[14,15]$. An example is MOSOCO [14] to help school children engage in sustained eye-contact and conversation by augmenting social situations with visual supports in the form of suggestions (e.g., "look at your partner's eyes").

Technological interventions deployed on mobile devices (i.e., smartphones, tablet PCs) reduce external barriers of accessibility and allow for the technology to be used in a variety of real-life situational contexts $[9,10]$. In a mobile AR application, teachers created a database of tagged images of objects for autistic children, who then used an object identification system in a school setting to identify them according to superimposed text and audio cues [15]. In addition to requiring minimal support while using the application, the children demonstrated a $45 \%$ increase in sustained attention during task completion [15].

Despite compelling evidence for the use of $A R$ as a supportive tool for childhood ASD interventions, there remains a lack of empirical evidence studying interventions for adults. Therefore, this paper aims to build a mobile AR-based self-help intervention which is more engaging and cost effective in addressing the barriers faced by the underserved population of autistic adults. Furthermore, it examines the usefulness of AR interventions in situational environments for adults outside lab and classroom settings. Given the potential of mobile AR applications to support individuals with ASD in real-world situations, we propose a Mobile Augmented Reality for Attention (MARA) tool. MARA uses AR technology as a means to improve attention in adults with ASD through simple interactive visual activities. 


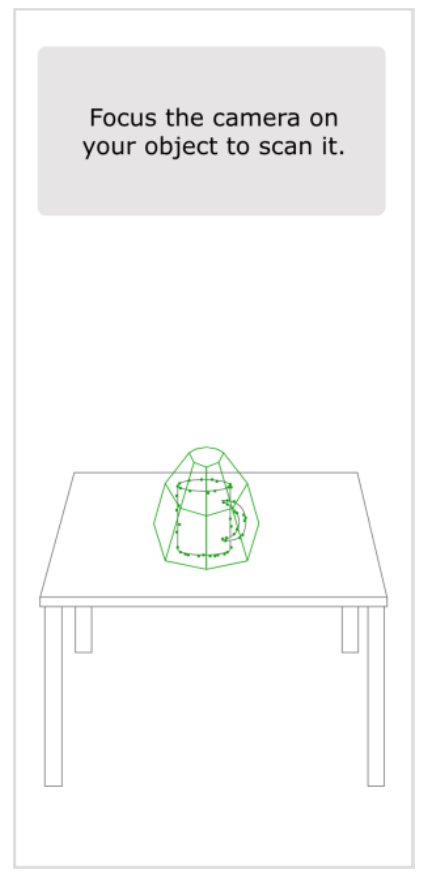

Figure 2: An illustration of the concept for selecting and scanning target object (e.g., coffee mug).

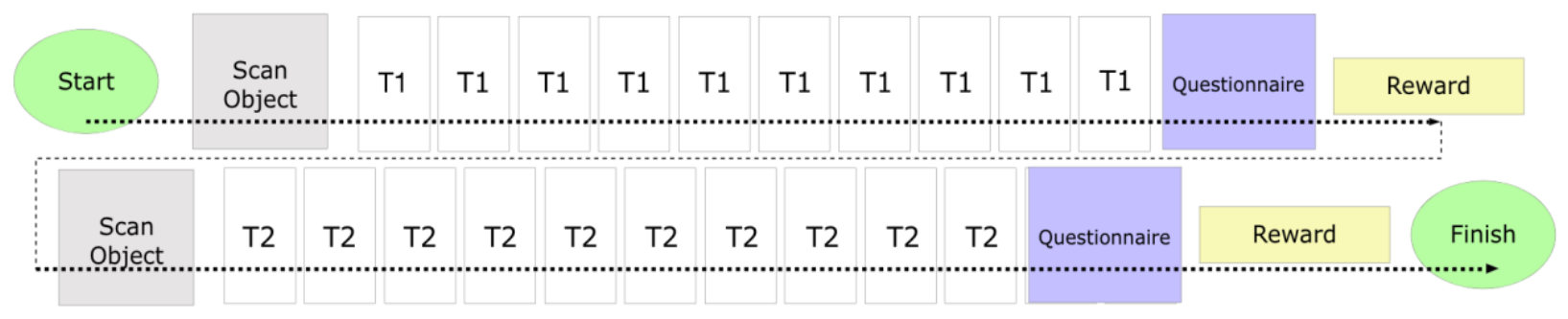

Figure 1: Proposed flowchart of the MARA therapy tool encountered by users once they have already completed the login requirements.

In the next section, we describe the design process of MARA, starting from our preliminary interviews for its conceptualization to the interface design. Also, we briefly discuss an experiment to test the efficacy of implementing the system as a therapy tool for attention impairments in adults with ASD.

\section{Proposed Design}

To obtain insight into how to conceptualize the MARA system, we conducted preliminary semi-structured interviews. Four healthy adults with ASD participated in the interviews (aged: 25-50 years, 4 males).

Participants highlighted the importance of keeping features as simple as possible given that many features in mobile applications are usually distracting and not easy to use. Also, P2 preferred not to seek "outside help" (e.g., therapists, institutions) to deal with his behaviors and was more interested in self-help methods. P3 reported on being prone to getting stuck in intrusive patterns of behavior and experiencing difficulties in stepping outside his own perception of reality. Lastly, all participants pointed out the lack of mobile applications suitable for helping autistic adults in any context (e.g., social, cognitive).
This has led to the first objective of developing MARA which is to determine preexisting therapies that help autistic individuals form new attention management behavior. One such method involves a blocking procedure, which has been evidenced to aid autistic children in acquiring the ability to discriminate between colors or numbers $[31,42]$. This procedure requires repeatedly differentiating between cards with different colors or digits with the aim of training the ability to readjust focus and discriminate between them. For instance, one study placed numbered cards in random locations on a table over ten trials and required children to match the correct numbered card to a target [31]. Several interventions also involved the use of prompts and rewards and found this elicited positive emotions and motivation afterwards $[15,31]$. The concept of MARA is modeled after these methods and involves integrating virtual stimuli with physical objects, prompting the user with repetitive tasks, and motivating them with rewards. Given these concepts, several interfaces were iteratively designed. With each iteration, tasks were also developed in an attempt to understand whether someone with attention deficits could intuitively use the interface and how repeatedly completing the activities could be used to alleviate these deficits. 


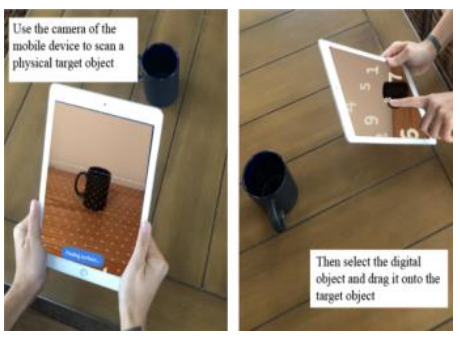

Figure 3: Testing the MARA interface using a coffee mug as the target object.

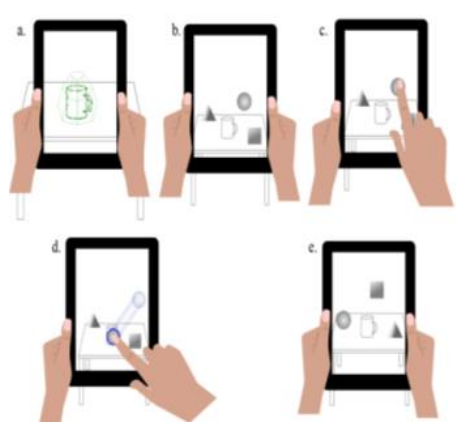

Figure 4: Performing a trial with the easy interface beginning with a. Target object scan; b. AR objects view; c. Object selection; d. Dragging the AR objects to target object; e. AR objects rearrange and tasks $b-d$ are repeated.
Lastly, we determined how goal-achievement would be addressed. There is evidence that reward achievement increases neural activity in regions associated with motivation and performance monitoring in autistic adults [35]. Users would therefore receive a reward compensation for using MARA (e.g. scores in [12]). Each time a session of ten trials and a post-task survey is completed, the reward feedback is displayed (see Figure 1). This amount is contingent on their performance (i.e., higher performance elicits a greater reward).

\section{Architecture}

The application interface is based on the Unity3D game engine and Vuforia, a mobile AR development kit that enables computer vision functionality on smartphone operating systems. The Vuforia prefabricated components used include the AR camera and ImageTarget for object tracking.

\section{Implementation}

Following informed consent, first-time users enter an activation code to log in and are prompted to fill out a multiple-choice demographic survey with questions regarding age, gender, type of ASD, age of diagnoses, other diagnosed disorders, history of misdiagnoses as well as a self-report sensory profile survey based off questions from the AASP and Autism-Spectrum Quotient [5]. Instructional videos on how to properly scan objects and use MARA are provided to the user along with a training session where they must successfully scan an object and complete one trial from each of the two sessions to demonstrate their understanding of the procedure. Results from this session are not recorded.
The application uses superimposition-based AR to directly overlay digital content onto a physical object using a smartphone. Figure 1 shows the flowchart of the system. Users encounter two sessions with ten sessions each. Each session begins with the user selecting an object in their surroundings they want to focus on (Figure 2). While the object itself does not need to be recognized, as it merely serves as a target, users receive positive feedback when different objects are scanned at the beginning of each session to motivate them to interact more with their environment. During the sessions, the view of the environment is partially replaced with an augmented view with virtual stimuli (Figure 3,4). After performing ten drag-anddrop tasks from the easy scenario, the user completes a short survey rating their levels of anxiety, motivation, and distraction on a visual analog scale. A rewards page displaying the user's total earnings appears following the self-assessment. The user then completes the next session (i.e., the difficult scenario) which is also followed by a questionnaire and earnings page.

Individuals with ASD are susceptible to extreme responses resulting from hyper-reactivity to sensory input [40], making it important to maintain a simple interaction model requiring minimal attention demand $[6,15]$. Furthermore, Biocca et al. [6] found mobile AR using visual cues provides greater decreases in cognitive load compared to other commonly used attentional techniques (e.g., highlighting). Taking this into consideration, we randomly selected three basic three-dimensional (3D) shapes to use during the easy scenario (see Figure 4). In the difficult scenario, nine virtual digits surround the target object (see Figure 5). During each session, a text box displayed at the top of the screen prompts users to drag and drop a specific 


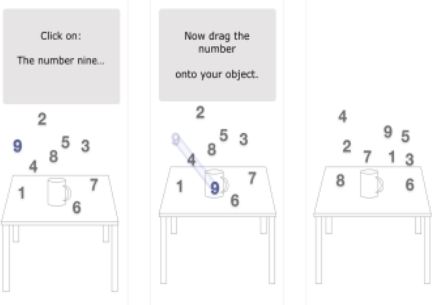

Figure 5: Step-by-step mobile interface concept for the 'difficult' condition digital object (i.e., shape or number) onto the target object. Once the user successfully does so, the game rearranges the virtual objects for the following task.

This is repeated ten times for each session. To avoid discriminating against those who may also suffer from other dysfunctions, such as color-blindness or dexterity impairments, designs with an overabundance of colorfu stimuli, as well as stimuli that moved around the screen were discarded.

User privacy and sensitive personal information gathered from surveys are taken seriously, therefore only limited access to encrypted data will be available to researchers and clinicians. Anonymous versions of quantitative and qualitative data can be accessed remotely through a secure, cloud-based database.

\section{Discussion and Future Work}

To address the external and internal barriers facing interventions for autistic adults, this paper has proposed a cost-effective self-help intervention using mobile AR. This demonstrates the ability for simple novel technologies to be used as effective therapy tools. It is also worth noting that MARA allows autistic adults to perform tasks independently, which supports a sense of autonomy critical to their integration into employment environments and social communities.

Key limitations of this short paper should be noted. First, while the design of MARA uses Vuforia as an AR development kit, Vuforia faces instabilities in tracking and stimuli recognition depending on environmental factors [41]. A possible solution involves testing the feasibility of MARA using Vuforia and addressing potential instability issues with alternative AR development or 3D mapping frameworks $[22,34]$.
Second, in order to enhance our understanding of the benefits of using MARA to train attention abilities, there is a need to involve experts on ASD to iteratively evaluate the system with studies involving participants with different ASD types. Further research is needed to discern how cognitive and physical abilities differ between high functioning adults and adults at the lower end of the spectrum in order to determine specific interventions appropriate for each type.

Lastly, several disorders that are highly comorbid with ASD share similarities in attention-related traits. For instance, approximately $40 \%$ of adults with ASD are also diagnosed with ADHD [19]. The presence of such confounding effects warrants further investigation on precise characteristics of attention. From there, we could explore additional interactive features (e.g. sound cues, haptic feedback), the integration of multimodal interactivity, as well as appropriate alterations to the proposed design. Addressing these limitations would help us identify more interesting ways to develop the application to make better use of AR to increase the user's interaction with their environment. A potential design, for instance, could incorporate the shape of the scanned object into the selection of virtual objects.

While the aim of the current paper was to identify the gaps in novel cost-effective interventions for adults with ASD, and to provide the first steps toward designing one, we expect to extend our preliminary research towards rigorous studies in which we develop and test a sustainable intervention solution to support a wider population of autistic adult and other neurodiverse groups, who struggle with attention management. 


\section{References}

[1] G. Allen and E. Courchesne. 2001. Attention function and dysfunction in autism. Frontiers in bioscience: a journal and virtual library 6: 105119.

[2] American Psychiatric Association. 2013. Diagnostic and Statistical Manual of Mental Disorders. American Psychiatric Association.

[3] Nancy Bagatell. 2016. The Routines and Occupations of Families With Adolescents With Autism Spectrum Disorders. Focus on Autism and Other Developmental Disabilities 31, 1: 49-59.

[4] Zhen Bai, Alan F. Blackwell, and George Coulouris. 2015. Using augmented reality to elicit pretend play for children with autism. IEEE Transactions on Visualization and Computer Graphics 21, 5: 598610.

[5] S Baron-Cohen, S Wheelwright, R Skinner, J Martin, and E Clubley. 2001. The autism-spectrum quotient (AQ): evidence from Asperger syndrome/high-functioning autism, males and females, scientists and mathematicians. Journal of autism and developmental disorders 31, 1: 5-17.

[6] Frank Biocca, Charles Owen, Arthur Tang, and Corey Bohil. 2007. Attention issues in spatial information systems: Directing mobile users' visual attention using augmented reality. Journal of Management Information Systems 23, 4: 163-184.

[7] Louanne E. Boyd, Alejandro Rangel, Helen Tomimbang, et al. 2016. SayWAT: Augmenting face-to-face conversations for adults with autism. Conference on Human Factors in Computing Systems - Proceedings: 4872-4883.

[8] Traolach S. Brugha, Sally McManus, John Bankart, et al. 2011. Epidemiology of Autism Spectrum Disorders in Adults in the Community in England. Archives of General Psychiatry 68, 5: 459.

[9] Youngjun Cho, Simon J. Julier, Nicolai Marquardt, and Nadia Bianchi-Berthouze. 2017. Robust tracking of respiratory rate in high-dynamic range scenes using mobile thermal imaging. Biomedical
Optics Express 8, 10: 4480.

[10] Youngjun Cho, Simon J Julier, and Nadia BianchiBerthouze. 2019. Instant Stress: Detection of Perceived Mental Stress Through Smartphone Photoplethysmography and Thermal Imaging. JMIR Mental Health 6, 4: e10140.

[11] Laura Crane, Lorna Goddard, and Linda Pring. 2009. Sensory processing in adults with autism spectrum disorders. 215-228.

[12] Tracy A. Dennis and Laura J. O'Toole. 2014. Menta Health on the Go. Clinical Psychological Science 2 5: 576-590.

[13] J. A. Easterbrook. 1959. The effect of emotion on cue utilization and the organization of behavior. Psychological Review 66, 3: 183-201.

[14] Lizbeth Escobedo, David H. Nguyen, Lou Anne Boyd, et al. 2012. MOSOCO: A mobile assistive tool to support children with autism practicing social skills in real-life situations. Conference on Human Factors in Computing Systems - Proceedings June 2014: 2589-2598.

[15] Lizbeth Escobedo, Mónica Tentori, Eduardo Quintana, Jesus Favela, and Daniel Garcia-Rosas. 2014. Using augmented reality to help children with autism stay focused. IEEE Pervasive Computing 13, 1: 38-46.

[16] A. Fridhi, F. Benzarti, A. Frihida, and H. Amiri. 2018. Application of Virtual Reality and Augmented Reality in Psychiatry and Neuropsychology, in Particular in the Case of Autistic Spectrum Disorder (ASD). Neurophysiology 50, 3: 222-228.

[17] Domingo García-Villamisar and C. Hughes. 2007. Supported employment improves cognitive performance in adults with Autism. Journal of Intellectual Disability Research 51, 2: 142-150.

[18] Sarah K. Howorth, Deborah Rooks-Ellis, Sara Flanagan, and Min Wook Ok. 2019. Augmented Reality Supporting Reading Skills of Students with Autism Spectrum Disorder. Intervention in School and Clinic 55, 2: 71-77. 
[19] Kate Johnston, Antonia Dittner, Jessica Bramham, Clodagh Murphy, Anya Knight, and Ailsa Russell.

2013. Attention Deficit Hyperactivity Disorder Symptoms in Adults with Autism Spectrum Disorders. Autism Research 6, 4: 225-236.

[20] Robert M. Joseph, Brandon Keehn, Christine Connolly, Jeremy M. Wolfe, and Todd S. Horowitz. 2009. Why is visual search superior in autism spectrum disorder? Developmental Science 12, 6: 1083-1096.

[21] Neha U. Keshav, Arshya Vahabzadeh, Rafiq AbdusSabur, et al. 2018. Longitudinal socio-emotional learning intervention for autism via smartglasses: Qualitative school teacher descriptions of practicality, usability, and efficacy in general and special education classroom settings. Education Sciences 8, 3 .

[22] Wasim Akram Khan, Dibakar Raj Pant, Bhisma Adhikari, and Rasana Manandhar. 2017. 3D object tracking using disparity map. 2017 International Conference on Computing, Communication and Automation (ICCCA), IEEE, 1015-1018.

[23] Meng-chuan Lai and Simon Baron-cohen. 2015. Identifying the lost generation of adults with autism spectrum conditions. 1013-1027.

[24] I. Jui Lee, Chien Hsu Chen, Chuan Po Wang, and Chi Hsuan Chung. 2018. Augmented Reality Plus Concept Map Technique to Teach Children with ASD to Use Social Cues When Meeting and Greeting. Asia-Pacific Education Researcher 27, 3: 227-243.

[25] Miriam Liss, Celine Saulnier, Deborah Fein, and Marcel Kinsbourne. 2006. Sensory and attention abnormalities in autistic spectrum disorders. Autism 10, 2: 155-172.

[26] Gonzalo Lorenzo, Marcos Gómez-Puerta, Graciela Arráez-Vera, and Alejandro Lorenzo-Lledó. 2019. Preliminary study of augmented reality as an instrument for improvement of social skills in children with autism spectrum disorder. Education and Information Technologies 24, 1: 181-204.
[27] Don D. McMahon, David F. Cihak, Rachel E. Wright and Sherry Mee Bell. 2016. Augmented reality for teaching science vocabulary to postsecondary education students with intellectual disabilities and autism. Journal of Research on Technology in Education 48, 1: 38-56.

[28] Hedda Meadan, Michaelene M. Ostrosky, Brooke Triplett, Amanda Michna, and Angel Fettig. 2011 Using Visual Supports with Young Children with Autism Spectrum Disorder. TEACHING Exceptional Children 43, 6: 28-35.

[29] Michiko Miyazaki, Tomohisa Asai, and Ryoko Mugitani. 2019. Touching! An augmented reality system for unveiling face topography in very young children. Frontiers in Human Neuroscience 13, June: 1-12.

[30] Behnaz Nojavanasghari, Charles E. Hughes, and Louis Philippe Morency. 2017. Exceptionally social: Design of an avatar-mediated interactive system for promoting social skills in children with autism. Conference on Human Factors in Computing Systems - Proceedings Part F1276: 1932-1939.

[31] Luis Antonio Pérez-González and Gladys Williams. 2002. Multicomponent Procedure to Teach Conditional Discriminations to Children With Autism. American Journal on Mental Retardation 107, 4: 293.

[32] Kathleen A. Quill. Instructional Considerations for Young Children with Autism: The Rationale for Visually Cued Instruction. Journal of Autism and Developmental Disorders 27, 6: 697-714.

[33] Iulian Radu, Katherine Guzdial, and Simina Avram. 2017. An observational coding scheme for detecting children's usability problems in augmented reality. IDC 2017 - Proceedings of the 2017 ACM Conference on Interaction Design and Children: 643-649.

[34] Diogo Santos Ortiz Correa and Fernando Santos Osorio. 2018. 3D Objects Recognition Using Artificial Neural Networks. 2018 XLIV Latin American Computer Conference (CLEI), IEEE, 288293. 
[35] Nicole Schmitz, Katya Rubia, Therese Van Amelsvoort, Eileen Daly, Anna Smith, and Declan G.M. Murphy. 2008. Neural correlates of reward in autism. British Journal of Psychiatry 192, 1: 19-24.

[36] Leann E Smith and Matthew J Maenner. 2012. Developmental Trajectories in Adolescents and Adults With Autism: The Case of Daily Living Skills. JAAC 51, 6: 622-631.

[37] Teodor Stefanut and Andreea Camelia Tripon. 2017. Educational Mobile Application using Sphero SPRK + in an Augmented Reality scenario.

Romanian Journal of Human - Computer

Interaction 10, 3: 231-249.

[38] James Tanaka, Audrey Bai, Jon Bowen, et al. 2015 From the small screen to the big world: mobile apps for teaching real-world face recognition to children with autism. Advanced Health Care Technologies: 37

[39] Julie Lounds Taylor and Marsha Mailick Seltzer. 2011. Employment and Post-Secondary
Educational Activities for Young Adults with Autism Spectrum Disorders During the Transition to

Adulthood. Journal of Autism and Developmental Disorders 41, 5: 566-574.

[40] Arshya Vahabzadeh, Neha U. Keshav, Rafiq AbdusSabur, Krystal Huey, Runpeng Liu, and Ned T. Sahin. 2018. Improved socio-emotional and behavioral functioning in students with autism following school-based smartglasses intervention: Multi-stage feasibility and controlled efficacy study. Behavioral Sciences 8, 10

[41] Jiwu Wang and Weixin Zeng. 2019. Research on the Realization Method of Augmented Reality based on Unity3D. Journal of Robotics, Networking and Artificial Life 6, 3: 195

[42] Gladys Williams, Luis Antonio Pérez-González, and Anna Beatriz Müller Queiroz. 2005. Using a Combined Blocking Procedure to Teach Color Discrimination to a Child with Autism. Journal of Applied Behavior Analysis 38, 4: 555-558. 